\title{
Fusaricidin Biosynthesis Is Controlled via a KinB-Spo0A-AbrB Signal Pathway in Paenibacillus polymyxa WLY78
}

\author{
Yunlong Li, Haowei Zhang, Yongbin Li, and Sanfeng Chen ${ }^{\dagger}$ \\ State Key Laboratory of Agrobiotechnology and College of Biological Sciences, China Agricultural University, Beijing, China
}

Accepted 20 August 2021.

Fusaricidins produced by Paenibacillus polymyxa are important lipopeptide antibiotics against fungi. The fusGFEDCBA (fusaricidin biosynthesis) operon is responsible for synthesis of fusaricidins. However, the regulation mechanisms of fusaricidin biosynthesis remain to be fully clarified. In this study, we revealed that fusaricidin production is controlled by a complex regulatory network including KinB-Spo0A-AbrB. Evidence suggested that the regulator AbrB represses the transcription of the fus gene cluster by direct binding to the fus promoter, in which the sequences (5'-AATTTTAAAATAAATTTTGTGATTT-3') located from -136 to -112 bp relative to the transcription start site is required for this repression. Spo0A binds to the $a b r B$ promoter that contains the Spo0A-binding sequences ( $5^{\prime}$-TGTCGAA-3', 0A box) and in turn prevents the further transcription of $a b r B$. The decreasing concentration of AbrB allows for the derepression of the fus promoter repressed by AbrB. The genome of $P$. polymyxa WLY78 contains two orthologs (named Kin1508 and Kin4833) of Bacillus subtilis KinB, but only Kin4833 activates sporulation and fusaricidin production, indicating that this kinase may be involved in phosphorylating Spo0A to initiate sporulation and regulate the abrB transcription. Our results reveal that Kin4833 (KinB), Spo0A, and AbrB are involved in regulation of fusaricidin production and a signaling mechanism that links fusaricidin production and sporulation.

Keywords: AbrB, bacteria-plant symbiosis, biocontrol, fusaricidin, genetics and gene regulation, KinB, molecular signaling, Paenibacillus polymyxa, regulation, secondary metabolism, Spo0A

Fusaricidins produced by Paenibacillus polymyxa are antifungal lipopeptide antibiotics (Haron et al. 2019; Jeong et al. 2019; Lee et al. 2013; Li and Chen 2019). Fourteen fusaricidin members,

\section{${ }^{\dagger}$ Corresponding author: S. Chen; chensf@cau.edu.cn}

Funding: This work was supported by the National Key Research and Development Program of China (grant no. 2017YFD0200807).

Nucleotide sequence data for kin1508, kin4833, spo0A, and $a b r B$ in $P$. polymyxa WLY78 are deposited in the GenBank database under accession numbers MT096381, MT096382, MT096380, and MT096379, respectively.

*The $e$-Xtra logo stands for "electronic extra" and indicates there are supplementary materials published online.

The author(s) declare no conflict of interest.

(c) (1) () $\odot$ Copyright $(92021$ The Author(s). This is an open access article distributed under the CC BY-NC-ND 4.0 International license. which contain a cyclic peptide (six amino-acids) and a common guanidinylated $\beta$-hydroxyl fatty acid chain, have been reported (Deng et al. 2011; Kajimura and Kaneda 1996; Reimann et al. 2017; Vater et al. 2015). Fusaricidins are synthesized by the products of the fusGFEDCBA operon (fusaricidin biosynthesis, fus) through a nonribosomal mechanism (Li and Chen 2019). Although fusaricidin has great potential use in medical and agricultural fields, only a few studies on the regulation mechanisms of fusaricidin biosynthesis are available. For example, mutation of $\mathrm{AbrB}$ resulted in increased fusaricidin levels and AbrB could directly bind to the fus promoter region in P. polymyxa SQR21 (Li et al. 2013) and the phosphoglucomutase gene obviously affected fusaricidin production in P. polymyxa E681 (Kim et al. 2014). Therefore, it is vital to fully understand the transcriptional regulation mechanisms of fusaricidin biosynthesis for enhancing its production.

Regulation mechanisms of lipopeptide antibiotics in Bacillus subtilis is well-studied. It is believed that some antibiotics, such as gramicidin $\mathrm{S}$, tryocidine, and surfactin were produced by B. subtilis around the onset of sporulation (Strauch et al. 2007). It has long been known that sporulation in B. subtilis is induced by nutrient limitation such as the relevant limiting growth substrates, sources of carbon, nitrogen, and phosphorus (Nandy and Venkatesh 2008). Spo0A is a global master regulator that governs the onset of stationary phase and sporulation (Stephenson and Hoch 2002). Spo0A binds to a specific DNA sequence $\left(5^{\prime}\right.$-TGTCGAA-3') known as the '0A box', where it acts as both a repressor of certain vegetatively expressed genes (e.g., $a b r B$ ) and an activator of genes involved in sporulation (Liu et al. 2003; Strauch et al. 1990). The acitivty of Spo0A is governed by a multicomponent phosphorelay, and phosphorylation of its $\mathrm{N}$ terminal at Asp55 could switch on the transcriptional regulation function of its $C$ terminal (Zhao et al. 2002). In Bacillus spp., the phosphorylation of Spo0A (Spo0A P) is controlled by five histidine kinases (KinA, KinB, KinC, KinD, and $\mathrm{KinE}$ ), each of which senses different signals of sporulation (Banse et al. 2011; Brunsing et al. 2005; Jiang et al. 2000; Zeriouh et al. 2014). Among these histidine kinases, KinA and $\mathrm{KinB}$ are thought to be the major kinases for initiating sporulation (Liu et al. 2018; Tojo et al. 2013).

AbrB is a transition state regulatory protein that represses the expression of many post-exponential phase functions, such as Spo0E, SpoOH, and SpoVG necessary for normal sporulation (Strauch et al. 1990). The AbrB protein is also involved in regulation of the expression of the genes for competence, antibiotics production, and biofilm formation in B. subtilis (Bochmann et al. 2015). For example, in B. subtilis biosynthesis of gramicidin $S$ is controlled by $\mathrm{SpoOA}, \mathrm{AbrB}$, and $\mathrm{SpoOH}$, and tyrocidine production is regulated by SpoOA and AbrB (Marahier et al. 1993), 
while surfactin biosynthesis is controlled by SpoOA and competencerelated gene products (ComA, ComP, and ComQ) required for the initiation of the competence developmental pathway (Fisher et al. 1994). It was reported that, in B. subtilis, Spo0A P represses AbrB and thereby AbrB decreases, allowing for the derepression of those promoters repressed by AbrB (Liu et al. 2003). Although AbrB regulates numerous genes in $B$. subtilis, it does not recognize a clear consensus DNA sequence, mainly due to the structural flexibility of AbrB (Olson et al. 2014).

Recently, we have reported that $P$. polymyxa WLY78 produces fusaricidin with strong antifungal activity against Fusarium species (Li and Chen 2019). Reverse transcription (RT)-PCR revealed that the seven genes (fus $G$, fus $F$, fus $E$, fusD, fus $C$, $f u s B$, and fusA) within the fus cluster responsible for fusaricidin biosynthesis of $P$. polymyxa WLY78 are organized as an operon. fusA is the largest gene, with a length of $23.73 \mathrm{~kb}$, which is responsible for synthesizing the cyclic polypeptide moiety of fusaricidin, while the other six genes (fusG, fusF, fusE, fusD, fus $C$, and $f u s B$ ) are responsible for synthesizing the lipid moiety of fusaricidin ( $\mathrm{Li}$ and Chen 2019).

In this study, mutation and complementation analyses reveal that $k i n 4833$ ( $k i n B), s p o 0 A$, and $a b r B$ are involved in regulation of fusaricidin biosynthesis. Electrophoretic mobility shift assays (EMSA) demonstrate that AbrB binds to the the fus promoter and Spo0A binds to the $a b r B$ promoter region. Furthermore, the putative AbrB-binding sequences in the fus promoter region is determined by truncating the fus promoter for promoter activity assay. To our knowledge, here is the first time that fusaricidin production has been revealed to be controlled by the KinB-Spo0AAbrB pathway. Our results will provide guidance for improving fusaricidin production by genetically modifying the regulatory network of the fus gene expression.

\section{RESULTS}

\section{Biological control of wheat Fusarium head blight.}

Wheat Fusarium head blight (also known as wheat scab) is a devastating factor to wheat production and food security. As described below, fusaricidin fermentation broth of $P$. polymyxa WLY78 was applied to the aerial part of wheat seedlings in field trials. The disease index and biocontrol efficacy were investigated. The fusaricidin fermentation broth showed $64 \%$ biocontrol efficacy against Fusarium head blight of wheat and improved yields by $24 \%$ (Fig. 1). The data suggest that fusaricidin antibiotic plays an important role in control of Fusarium head blight.

\section{Disruption of the $a b r B$ gene leads to overproduction of fusaricidin.}

In order to enhance fusaricidin production, we investigated the transcriptional regulation mechanisms of fusaricidin biosynthesis. It is known that the AbrB protein is involved in regulation of antibiotics production in B. subtilis (Bochmann et al. 2015). In this study, we find that the 84-residue AbrB of $P$. polymyxa WLY78 exhibits $61 \%$ identity with AbrB (AQR84191.1) of B. subtilis 168 at the amino-acid sequence level and, also, that the DNA-binding domain is conserved (Supplementary Fig. S1). To clarify whether AbrB regulates the transcription of the fus operon, we constructed a $\triangle a b r B$ mutant. The antifungal activity against Fusarium asiaticum of $\triangle a b r B$ mutant was much stronger than that of wildtype $P$. polymyxa WLY78 (Fig. 2A). Quantitative (q)RT-PCR analysis showed that the transcript level of the fus $A$ gene in the $\triangle a b r B$ mutant was significantly higher than that in the wild-type strain, suggesting that $\mathrm{AbrB}$ negatively regulates transcription of the fus operon (Fig. 2B). To further confirm the negative regulation of AbrB on fus expression, a Pfus 3 -gfp plasmid carrying the entire fus promoter region (from -336 to +26 relative to the transcription start site) fused to the $g f p$ (green fluorescent protein) coding region was introduced into the $\triangle a b r B$ mutant and wildtype strain, respectively. As shown in Figure 2C, the GFP expression level in $\triangle a b r B$ mutant was almost onefold higher than that in the wild-type strain, suggesting that mutation in the $a b r B$ gene relieved the inhibition of AbrB on the fus transcription. All these results demonstrated that AbrB negatively regulates fus expression, consistent with the results of higher fusaricidin accumulation in $\triangle a b r B$ mutant of $P$. polymyxa SQR-21 ( $\mathrm{Li}$ et al. 2013).

\section{Determination of the transcription start site} and the AbrB-binding sequences in the fus promoter.

Our recent study has shown that the seven genes (fus $G, f u s F$, fus $E$, fus $D$, fus $C$, fus $B$, and fusA) within the fus cluster responsible for fusaricidin biosynthesis of $P$. polymyxa WLY78 are organized as an operon (Li and Chen 2019). In this study, the transcriptional start site (TSS) of the fus cluster is determined by using the $5^{\prime}$ RACE (rapid amplification of cDNA ends) method. The TSS is adenine located 26 bp upstream of the translation start codon (ATG) of fus $G$ and a putative ribosome-binding site is identified $8 \mathrm{nt}$ preceding the start codon. The -35 (TAGACA) and -10 (TATTTT) sequences are found in the fus promoter (Fig. 3A).

To ascertain whether AbrB protein binds to the fus promoter, the $P$. polymyxa $\mathrm{AbrB}$ protein with a $\mathrm{His}_{6}$-tag was overexpressed and was purified in Escherichia coli BL21. The purified AbrB is a $13-\mathrm{kDa}$ protein in sodium dodecyl sulfate-polyacrylamide gel electrophoresis (SDS-PAGE) (Fig. 3B). The entire fus promoter fragment (362 bp) was synthesized and used for assaying the interaction between the fus promoter and AbrB. EMSA revealed the binding interaction between $\mathrm{AbrB}$ and the fus promoter (Fig. 3C). The binding is specific, as a DNA fragment of similar length derived from the fusA coding sequence showed no binding affinity to AbrB (Supplementary Fig. S2).

AbrB does not recognize a single consensus sequence in $B$. subtilis (Strauch et al. 1989). AbrB binds a number of DNA targets with a flexible structure, which allows a conformational change when AbrB binds (Bobay et al. 2004). Usually, DNA binding sites of transcription factors can be predicted by using the MEME/MAST program (Bailey et al. 2009). However, we failed to find consensus sequences in the AbrB-binding site of the fus promoter of $P$. polymyxa WLY78 by using the MEME/MAST method, suggesting that the $P$. polymyxa AbrB binds to a flexible structural DNA. Thus, we searched the AbrB-binding sequences by truncating the fus promoter region. Five different lengths of the fus promoter region (Pfus) fused to the $g f p$-coding region were cloned to vector $\mathrm{pHY} 300 \mathrm{PLK}$, respectively, yielding plasmids Pfus3-gfp, Pfus2-gfp, Pfusl-gfp, Pfus0-gfp, and Pfus--gfp. The five fus promoter regions include a common downstream region ( +26 bp relative to the transcription start site) and various upstream sequences from $-336 \mathrm{bp},-236 \mathrm{bp},-136 \mathrm{bp},-36 \mathrm{bp}$, and -1 bp relative to the transcription start site, respectively. Then, the five plasmids were individually introduced into $P$. polymyxa WLY78 and the $\triangle a b r B$ mutant to compare their expression profiles. As shown in Figure 3D, the PfusO-gfp and Pfus ${ }^{-}$- $g f p$ could not make both $P$. polymyxa WLY78 and $\Delta a b r B$ mutant exhibit GFP activities, mainly due to no promoter region $\left(\mathrm{P} f u s^{-}\right)$or the promoter region being too short (PfusO) for RNA polymerase function. In contrast, Pfus3-gfp, Pfus2-gfp, and Pfus 1-gfp enabled both $P$. polymyxa WLY78 and $\triangle a b r B$ mutant to exhibit GFP activities. The results suggest the possible location of an AbrB-binding site in the 136-bp promoter region of the fus gene.

In order to determine the putative AbrB-binding site in the fus promoter, we further constructed three additional plasmids Pfus0.75-gfp, Pfus0.5-gfp, and Pfus0.25-gfp, each of which individually carries a different length of the fus promoter region (from $-111,-86$, and -61 bp to +26 bp relative to the 
transcription start site, respectively) fused to the $g f p$ coding region. Then, these plasmids together with plasmid Pfusl-gfp were respectively introduced into the recombinant $E$. coli BL21, which carries the $P$. polymyxa $a b r B$ gene under the control of the T7 promoter in pET-28b vector. As shown in Figure $3 \mathrm{E}$, in the absence of an isopropyl- $\beta$-D-thiogalactoside (IPTG)-induction (-) group, Pfusl-gfp, Pfus0.75-gfp, Pfus0.5-gfp, and Pfus0.25-gfp each showed high GFP activity with no significant difference among them, indicating that these fus promoters were barely regulated, consistent with lack of the expressed AbrB. When IPTG was added (+), these $g f p$-fusion plasmids (Pfus0.75-gfp, Pfus0.5-gfp, and Pfus0.25-gfp) stably expressed high GFP activity, while Pfus l-gfp showed very low GFP activity. The data suggested that the 25-bp DNA region from -136 to -112 bp relative to the transcription start site is required for the AbrB binding. The regulator AbrB represses the transcription of

A

WLY78

Control
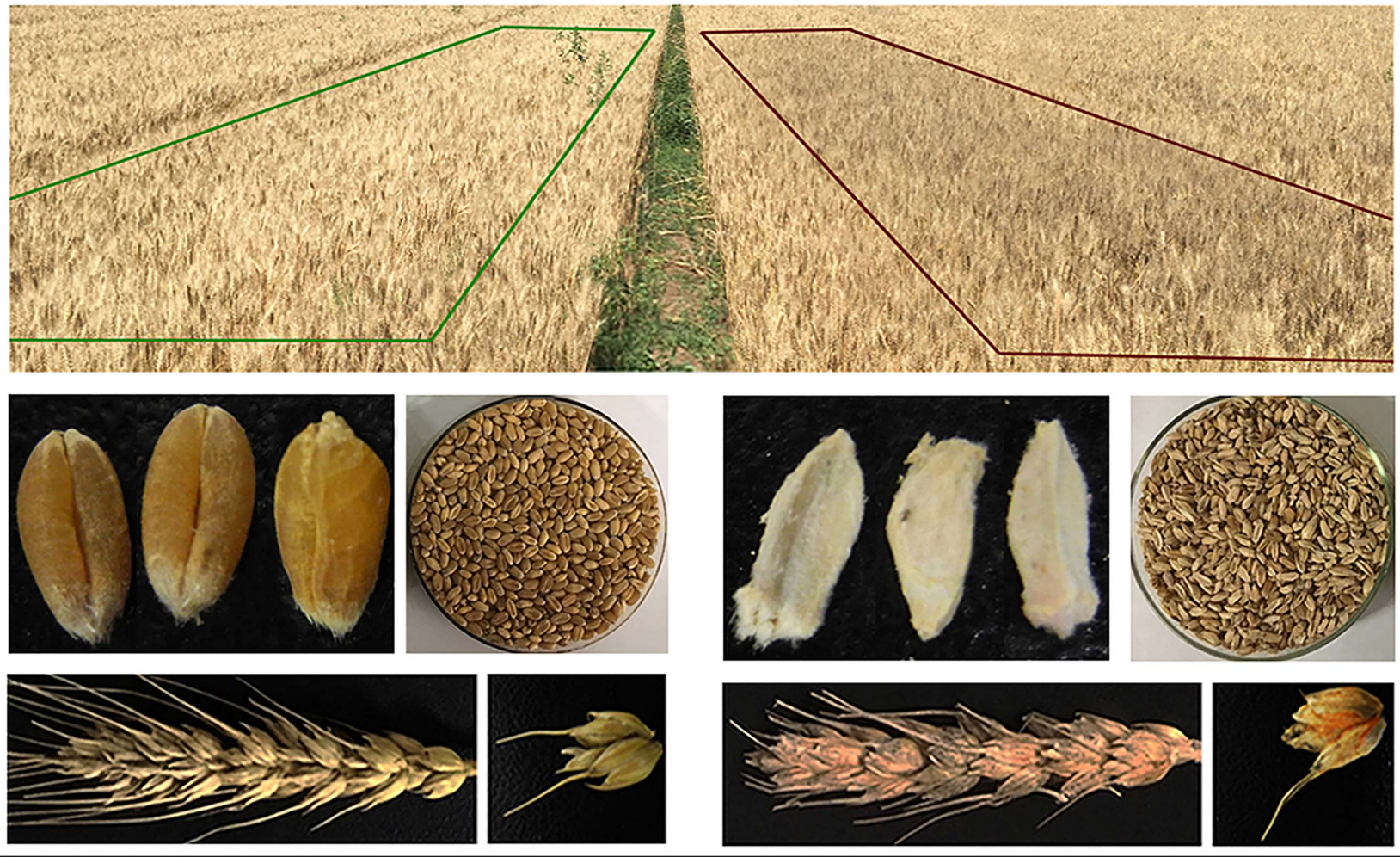

B

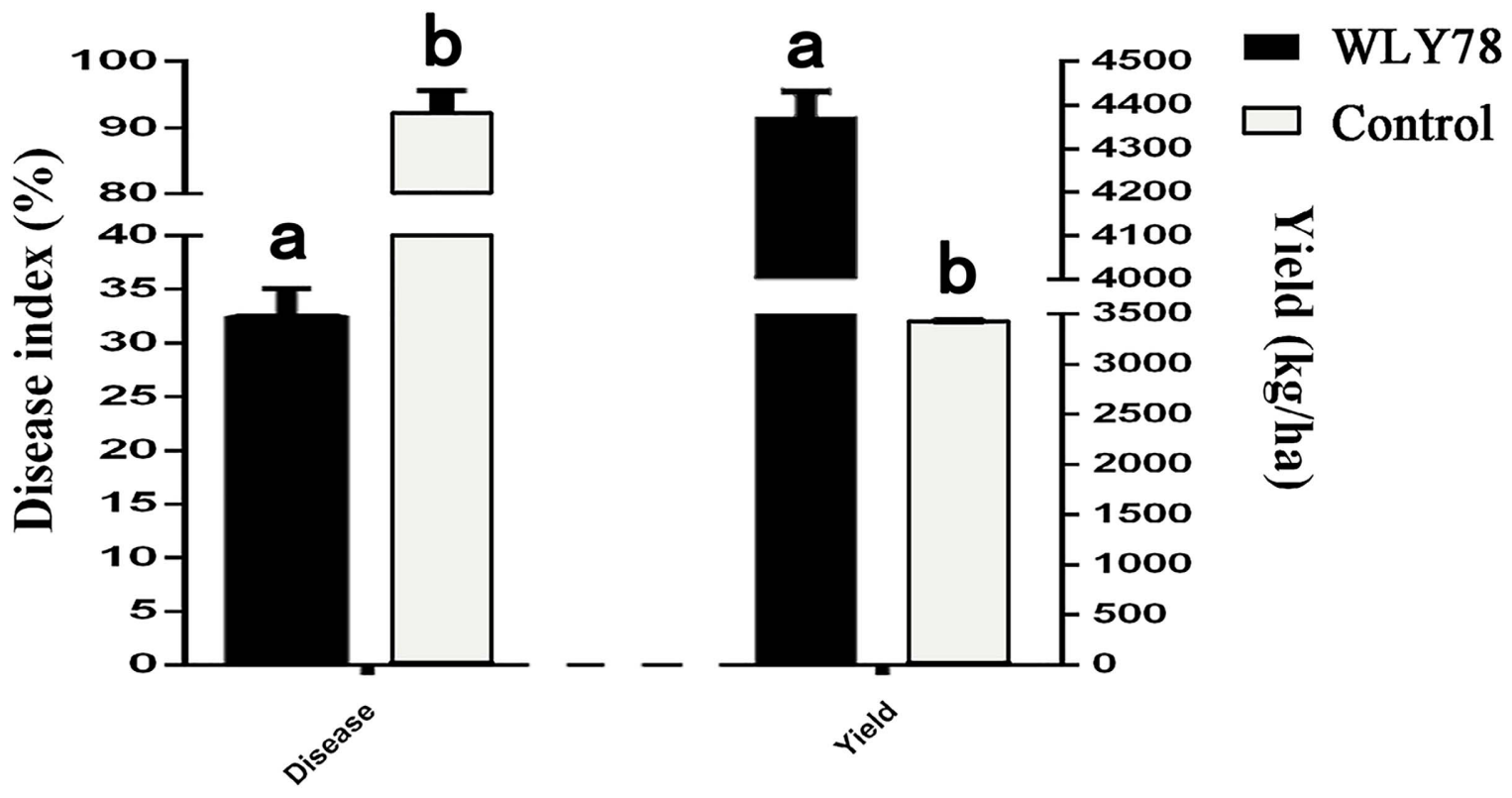

Fig. 1. Biological control of wheat Fusarium head blight with fusaricidin fermentation broth. A, Comparison of disease symptoms of wheat plants and grains in fields between treatments with fusaricidin fermentation broth of Paenibacillus polymyxa WLY78 (designated WLY78) and with tap water (control). B, Comparison of disease index and wheat yield in fields between treatments with fusaricidin fermentation broth (designated WLY78) and with tap water (control). Lower case letters indicate statistically significant difference $(P<0.05)$. 
the fus gene cluster, likely by direct binding to the fus promoter, in which the sequences (5'-AATTTTAAAATAAATTTTGT GATTT-3') located from -136 to -112 bp relative to the transcription start site is required for this repression (Fig. 3F). The 25-bp site in the fus promoter region is rich in $\mathrm{A}+\mathrm{T}$ sequences. The AbrB-binding sequences that are rich in $\mathrm{A}+\mathrm{T}$ content are reported in the promoter regions of B. subtilis spoOH, spoOH, kinB, ftsA, and pbpE (Frisby and Zuber 1991; Strauch 1995a). The B. subtilis spoOH promoter region has the AbrB-binding sequences (AATATGTTTACAAATAAAGTATAATCTGTA ATAATGCAC) and the B. subtilis $a b r B$ promoter region has the AbrB-binding sequences (ACAAAATGATTGACGAT TATTGGAAACCTTG) (Strauch 1995b). It is believed that A+T-rich sequences create a "bent" conformation in the DNA and the bent conformation may help to open the DNA helix, which could enhance the binding of RNA polymerase to the promoter by allowing additional polymerase-DNA contacts to occur (Frisby and Zuber 1991).

\section{Spo0A positively regulates the fus expression} by repressing $a b r B$ gene transcription.

$P$. polymyxa WLY78 contains a spo0A gene whose predicted product Spo0A (267 amino acids) exhibits 69\% identity with Spo0A (CAB14353.1) of B. subtilis. Moreover, the N-terminal phosphorylation site Asp55 and the C-terminal helix-turn-helix DNA-binding domain are found to be conserved in both $P$. polymyxa Spo0A and B. subtilis Spo0A (Supplementary Fig. $\mathrm{S} 3$ ). To determine whether Spo0A of $P$. polymyxa WLY78 influences fusaricidin production, spoOA deletion mutant ( $\triangle$ spo0A) was constructed. Compared with P. polymyxa WLY78, the $\triangle$ spoOA mutant completely lost the antifungal activity against $F$. asiaticum (Fig. 4A), indicating that $P$. polymyxa Spo0A is required for fusaricidin production. Also, the $\triangle$ spoOA mutant completely lost the ability to form spores (Fig. 4B), suggesting the correlation between sporulation and fusaricidin production. Furthermore, qRT-PCR analysis revealed that the transcription level of the fusA gene in the wild-type strain was 10-fold higher than that in $\triangle$ spoOA mutant (Fig. 4C). Meantime, the Pfus3-gfp plasmid, which carries the entire fus promoter region fused to the gfp coding region, was introduced into the wild-type strain and the $\triangle$ spoOA mutant, respectively. As shown in Figure 4D, the GFP expression level in wild-type strain was almost 15 -fold higher than that in $\triangle$ spoOA mutant. All these data indicate that Spo0A positively affects fusaricidin production via transcriptional regulation.

To examine whether Spo0A directly binds to the promoter region of the fus operon, we overexpressed and purified the $P$. polymyxa Spo0A with $\mathrm{His}_{6}$-tag in $E$. coli $\mathrm{BL} 21$, and the Spo0A protein has a deduced molecular weight of $33.5 \mathrm{kDa}$ in SDS-PAGE (Fig. 5A). EMSA experiments revealed that Spo0A didn't bind to the fus promoter region (Fig. 5B), indicating that the Spo0A indirectly regulates the fus transcription. The results are consistent with the finding that the fus promoter region does not contain any 0A boxes (TGTCGAA). Then, we searched and found there are two 0A boxes (TGTCGAA) in the $a b r B$ promoter regions of $P$. polymyxa WLY78 (Fig. 5C). The EMSA
A
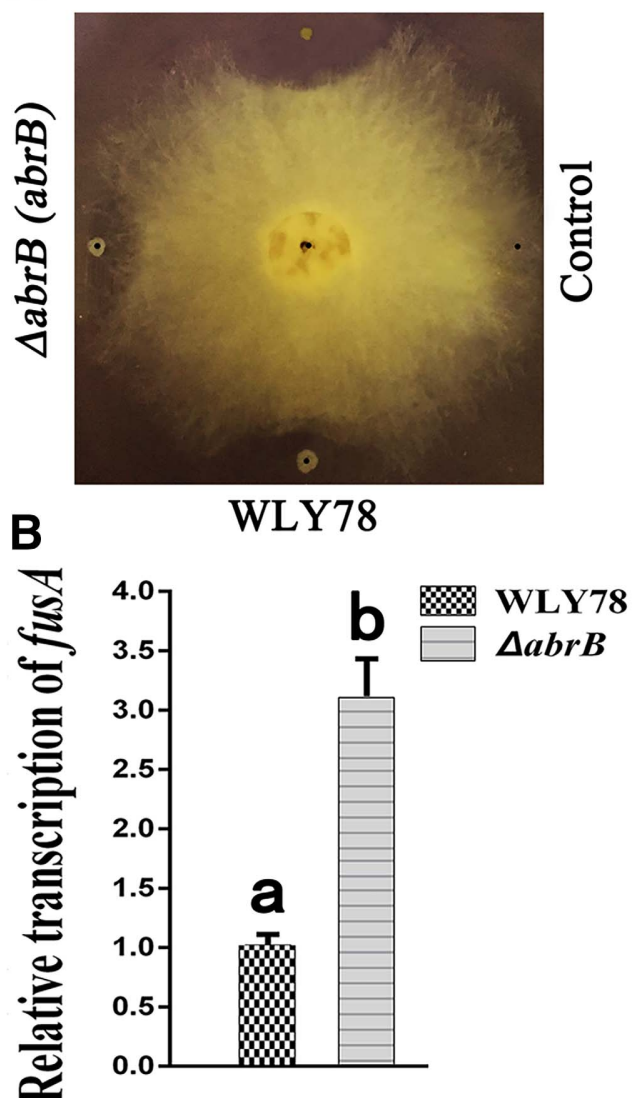

C
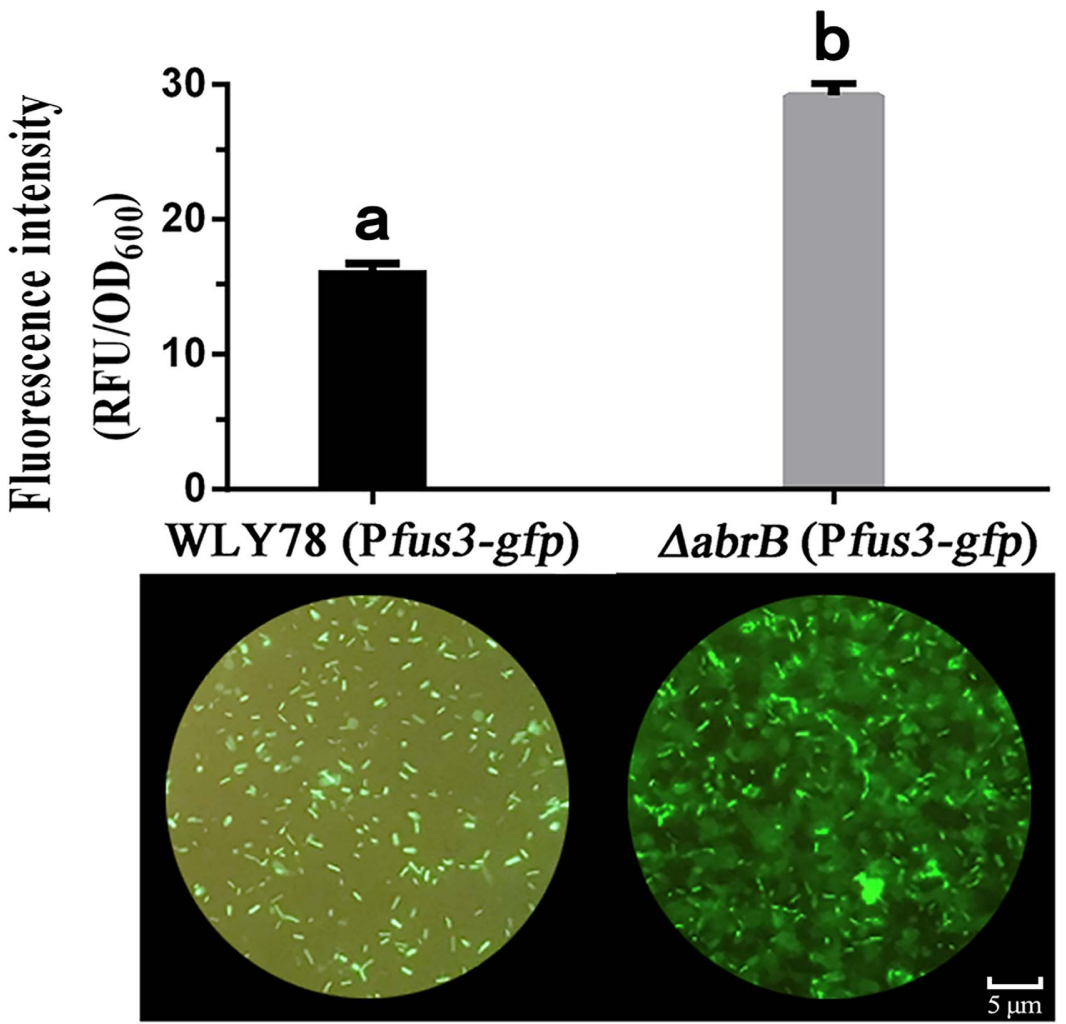

Fig. 2. Comparison of the antifungal abilities, transcription levels of the fusA gene, and fluorescence intensities of the Pfus3-gfp fusion among Paenibacillus polymyxa WLY78, $\triangle a b r B$ mutant, and complementation strain $\triangle a b r B(a b r B)$. A, The inhibition activities against Fusarium asiaticum by $P$. polymyxa WLY78, $\triangle a b r B$ mutant, and complementation strain $\Delta a b r B(a b r B)$, with sterilized water as control. B, Transcription levels of the fusA gene in $P$. polymyxa WLY78 and $\triangle a b r B$ mutant determined by quantitative reverse transcription PCR. C, Fluorescence microscope observation and fluorescence intensities of in $P$. polymyxa WLY78 and $\Delta a b r B$ mutant carrying the Pfus3-gfp fusion. Lower case letters indicate statistically significant difference $(P<0.05)$. 
A

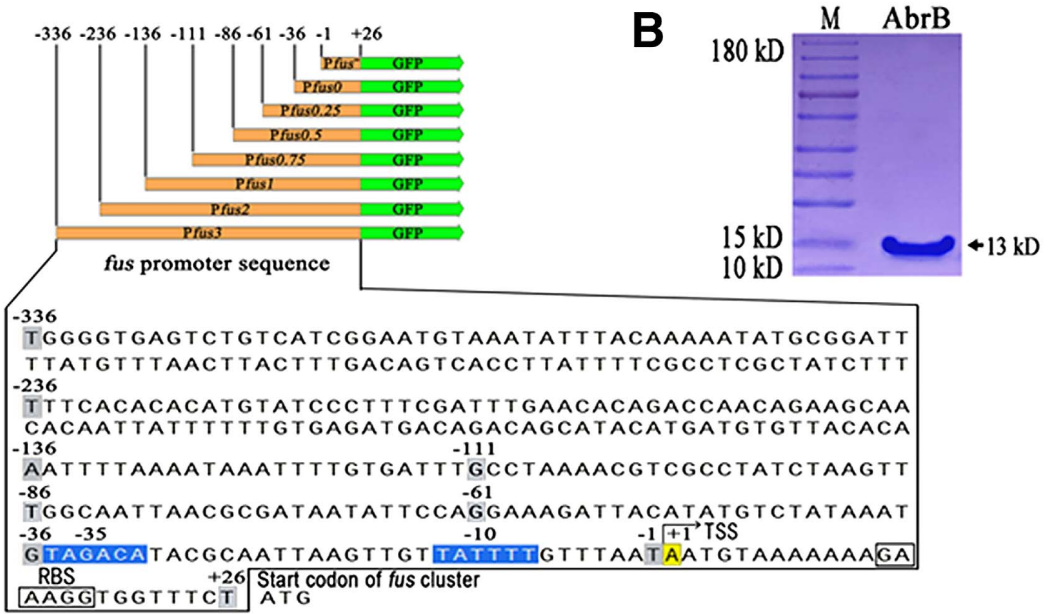

D

E

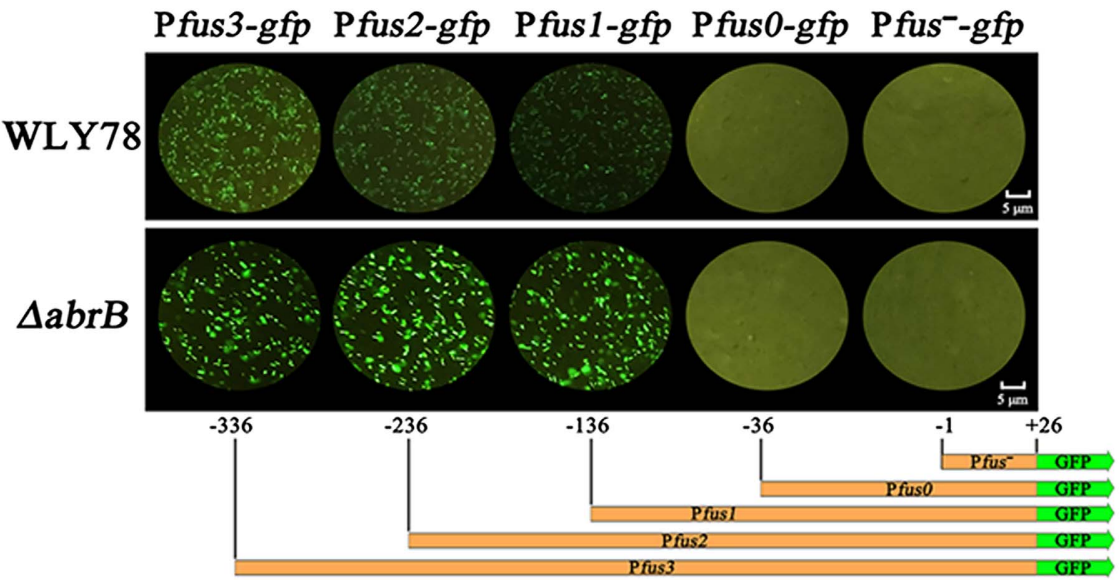

\section{IPTG Pfus1-gfp Pfus0.75-gfp Pfus0.5-gfp Pfus0.25-gfp}

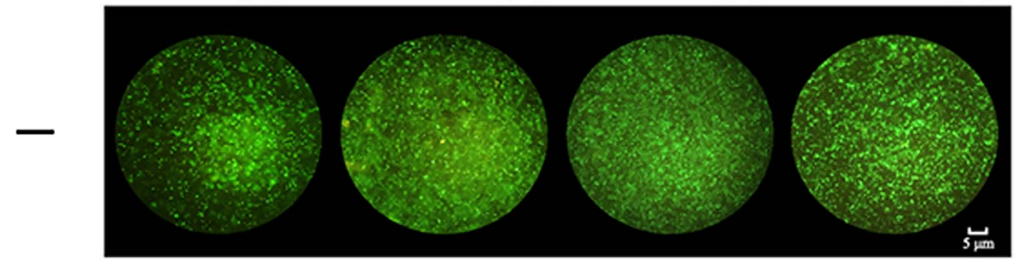

$\mathbf{F}$

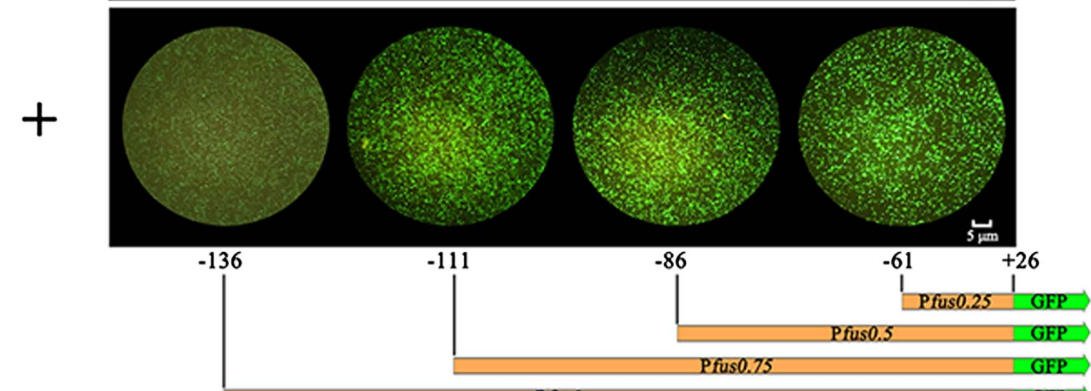

C
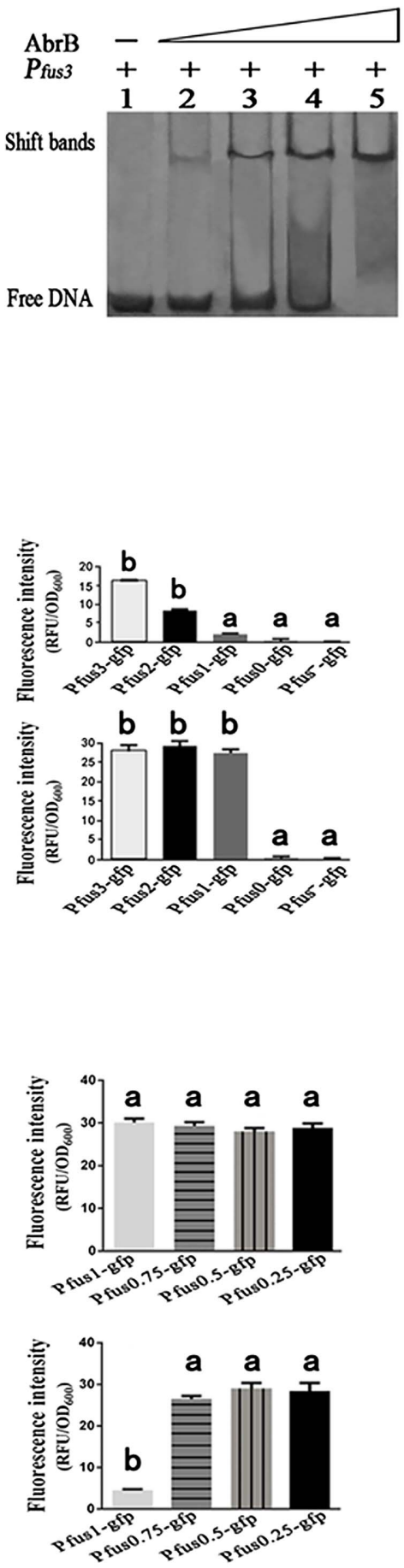

Fig. 3. Determination of AbrB-binding site in the fus promoter by electrophoretic mobility shift assay (EMSA) and by assaying the fluorescence intensities of the different Pfus-gfp fusions in Paenibacillus polymyxa WLY78, the $\Delta a b r B$ mutant, and Esherichia coli. A, Map of the different Pfus-gfp fusions (Pfus ${ }^{-}$-gfp, Pfus0-gfp, Pfus0.25-gfp, Pfus0.5-gfp, Pfus0.75-gfp and Pfus1-gfp, Pfus1-gfp, Pfus2-gfp and Pfus3-gfp). B, Sodium dodecyl sulfatepolyacrylamide gel electrophoresis analysis of the expressed and purified P. polymyxa His $_{6}-$ AbrB protein in E. coli. C, EMSA analysis of the binding ability of AbrB protein to the fus promoter region. Lanes 1 through 5 indicate increasing concentrations of AbrB protein $(0,5,20,50,200 \mathrm{ng})$ and 0.1 pmol-labeled promoter DNA was used for each lane. D, Fluorescence microscope observation and fluorescence intensities of $P$. polymyxa WLY78 and $\triangle a b r B$ mutant carrying the different Pfus-gfp fusions. E, Fluorescence microscope observation and fluorescence intensities of the recombinant $E$. coli BL21 carrying the $a b r B$ gene in the pET-28b vector, which allows the expression of $P$. polymyxa AbrB protein when isopropyl- $\beta$-D-thiogalactoside (IPTG) is present and carrying the different Pfus-gfp fusions. The "+" and "-" symbols indicate that IPTG was supplied or not, respectively. F, The AbrB-binging site located from -136 to -112 bp relative to transcription start site of the fus promoter. Lower case letters indicate statistically significant difference $(P<0.05)$. 
experiments demonstrated that Spo0A directly bind to the $a b r B$ promoter region (Fig. 5D). qRT-PCR revealed that the transcript levels of the $a b r B$ gene in $\triangle s p o O A$ mutant were onefold higher than that in wild-type strain (Fig. 5E). The results suggest that $P$. polymyxa Spo0A represses $a b r B$ transcription, consistent with the findings that Spo0A negatively controlled $a b r B$ expression in B. subtilis 168 (Fujita et al. 2005; Strauch et al. 2007). Therefore, $P$. polymyxa Spo0A indirectly activates fusaricidin production by directly repressing $a b r B$ expression. Our findings support the reports that spontaneous asporogenic mutants of $P$. polymyxa do not produce antibiotics (polymyxin) and support the dependence of polymyxin production on sporulation (Kaur et al. 1978; Nefelova et al. 1980; Park et al. 2012).

\section{Kin4833 (KinB) functions upstream of Spo0A and} activates sporulation and fusaricidin production.

It was reported that, in B. subtilis, either KinA or KinB is the sensor kinase that carries out auto-phosphorylation at a histidine residue and then transfers a phosphoryl group to an aspartyl residue in $\mathrm{Spo} 0 \mathrm{~F}$, and the resulting $\mathrm{Spo} 0 \mathrm{~F} \sim \mathrm{P}$, in turn, transfers the phosphoryl group to Spo0B. Finally, Spo0B $\sim \mathrm{P}$ transfers the phosphoryl group to Spo0A and the resulting Spo0A $\sim \mathrm{P}$ activates or represses gene expression (Perego et al. 1989; Tojo et al. 2013).

In this study, we screened the $P$. polymyxa WLY78 genome with the conserved histidine kinase A phosphoacceptor (HisKA) domain of B. subtilis histidine kinases and found two putative histidine kinases, named Kin1508 and Kin4833. Comparison of $P$. polymyxa Kin1508 and Kin4833 with B. subtilis KinA, KinB, $\mathrm{KinC}$, KinD, and KinE at the amino-acid sequence level showed that the conserved residues, especially a histidine residue for auto-phosphorylation, exist among all the proteins (Fig. 6A). Further, protein domain analysis showed that each of the Kin1508 and Kin4833 proteins of $P$. polymyxa WLY78 has three domains, transmembrane, HisKA, and ATPase. The phylogenetic tree shows that Kin1508 and Kin4833 of P. polymyxa WLY78 have relate closely with KinB of B. subtilis 168 (Fig. 6B). Moreover, phylogenetic analysis of Kin 1508 and Kin4833 demonstrates their high homology to KinB by aligning them with all the published histidine kinase nucleotide sequences from Bacillus spp. (Supplementary Fig. S4). The identity of the histidine kinase nucleotide sequences is $41.23 \%$ (Supplementary Fig. S5). Similar analysis shows that $P$. polymyxa Kin1508 and Kin4833 exhibit 23 and $31 \%$ identity, respectively, with KinB of B. subtilis 168, at the amino-acid sequence level (Supplementary Fig. S6). B. subtilis KinB was reported to phosphorylate Spo0A to initiate sporulation (Liu et al. 2018). Thus, we deduce that $P$. polymyxa WLY78 Kin1508 and Kin4833 may phosphorylate Spo0A just as B. subtilis $\mathrm{KinB}$ did.

To further determine whether or not Kin1508 and Kin4833 are involved in phosphorylation of Spo0A, two deletion mutants,
A

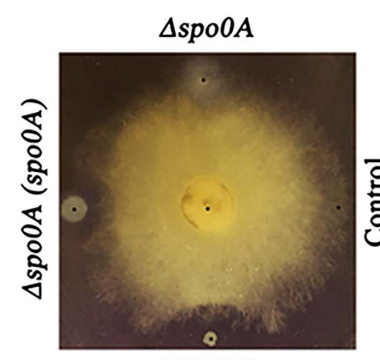

WLY78
C

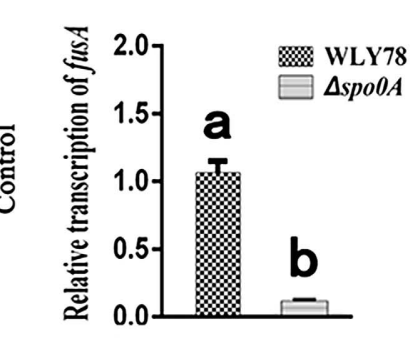

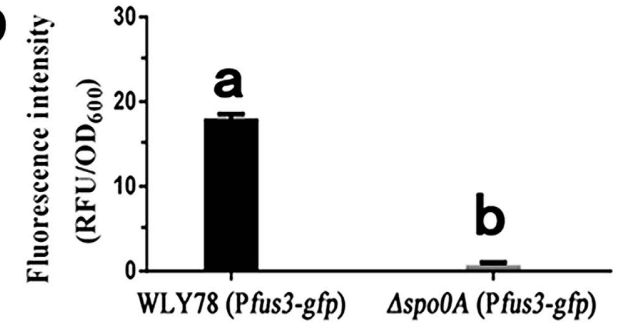

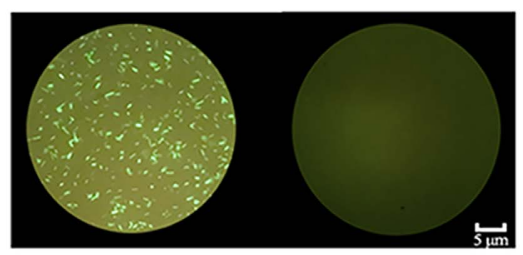

B

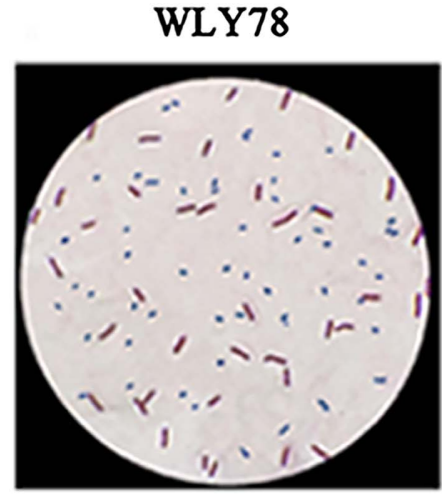

$60 \%$ sporulation $\triangle$ spo0A

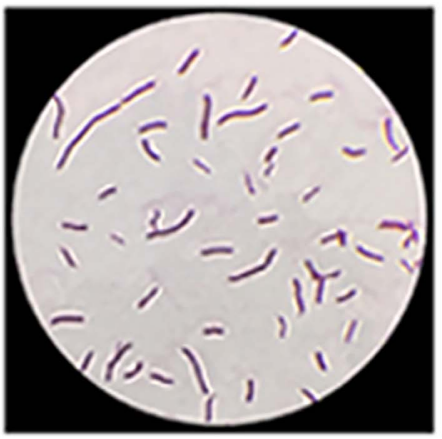

$0 \%$ sporulation $\triangle \operatorname{spo} 0 A(\operatorname{spo} 0 A)$

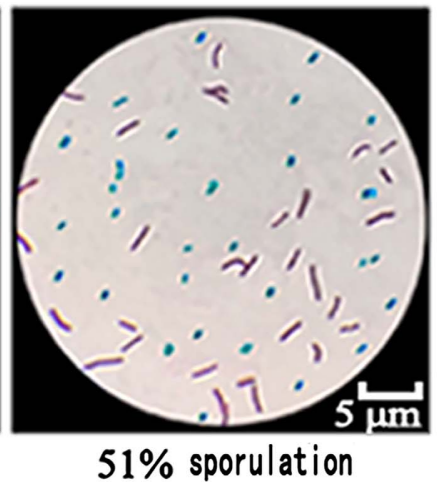

Fig. 4. Comparison of the antifungal abilities, endospore formation, transcription levels of the fusA gene and fluorescence intensity of the Pfus3-gfp fusion among Paenibacillus polymyxa WLY78, $\triangle$ spoOA mutant, and complementation strain $\triangle$ spoOA (spoOA). A, The inhibition activities against Fusarium asiaticum by $P$. polymyxa WLY78, $\triangle$ spo0A mutant, and its complementation strain $\triangle$ spoOA (spo0A). B, Sporulation frequencies of $P$. polymyxa WLY78, $\triangle$ spoOA mutant, and complementation strain $\triangle$ spoOA (spoOA). Rod cell in dark red is vegetative cell and spheroid cell in blue is spore. C, Transcription levels of the fusA gene in P. polymyxa WLY78 and $\triangle$ spoOA mutant determined by quantitative reverse transcription-PCR. D, Fluorescence microscope observation and fluorescence intensity of $P$. polymyxa WLY78 and $\Delta$ spo0A mutant carrying the Pfus3-gfp fusion. Lower case letters indicate statistically significant difference $(P<0.05)$. 
$\Delta$ kin1508 and $\Delta$ kin4833, as well as their complementary expression $\Delta$ kin1508 (pHY-Pkin1508) and $\Delta$ kin4833 (pHY-Pkin4833) were constructed. Here, the sporulation frequencies are used as an indicator of the phosphorylation levels of Spo0A (Spo0A P), based on reports that $B$. subtilis initiated sporulation only if a threshold concentration of phosphorylated Spo0A was reached (Kiehler et al. 2017). Compared with wild-type $P$. polymyxa WLY78, $\Delta$ kin1508 mutant showed no obvious difference in sporulation efficiency, but the $\Delta$ kin 4833 mutant showed significant reduction in sporulation ability. Complementation of the $\Delta$ kin4833 mutant with kin4833 restored the sporulation defect, indicating that Kin4833 may be involved in phosphorylation of Spo0A to initiate sporulation (Fig. 6C).

To elucidate the effect of Kin4833 on fusaricidin production, the antifungal activities against $F$. asiaticum of $\Delta$ kin 4833 mutant, complementation strain $\Delta k i n 4833$ (pHY-kin4833), and the wild-type strain were detected. The inhibition zone of $\Delta$ kin 4833 is obviously smaller than that of $P$. polymyxa WLY78, while complementation strain $\Delta$ kin4833 (pHY-kin4833) rescues the antifungal effect of mutant $\Delta$ kin4833 (Fig. 6D). These results indicate, for the first time, that kin4833 positively regulates fusaricidin production. To examine the effect of $\Delta$ kin 4833 mutation on the transcription of the fus operon of $P$. polymyxa WLY78, the transcription levels of the fusA gene were determined by qRT-PCR. The transcription level of the fusA gene in the wild-type strain exhibited threefold higher than that in $\Delta$ kin4833 mutant (Fig. 6E). This result is consistent with an antifungal phenotype of $\Delta$ kin 4833 mutant, indicating that Kin4833 (KinB) positively regulates the fus transcription.
To further confirm the effect of Kin4833 on regulation of the fus expression, a Pfus3-gfp plasmid carrying the entire fus promoter region fused to the $g f p$ coding region was introduced into wild type and $\Delta \operatorname{kin} 4833$ mutant, respectively. As shown in Figure $6 \mathrm{~F}$, the GFP expression level in the wild-type strain was almost fourfold higher than that of $\Delta$ kin 4833 mutant, consistent with the antifungal activity and the fusA transcription level. Overall, our results demonstrate that the fusaricidin production and sporulation are both activated by Kin4833 (KinB). Our results are consistent with the reports that high amounts of fusaricidin was produced only when cells entered into sporulation (Beatty and Jensen 2002).

\section{DISCUSSION}

Fusarium head blight has caused serious damage to wheat production in China. Multidisciplinary efforts have been made to fight against this disease for a long time (Ma et al. 2020). Our previous studies revealed that the fusaricidin antibiotic produced by $P$. polymyxa WLY78 plays an important role in inhibition against Fusarium species, Verticillium albo-atrum, and other plant-pathogenic fungi ( $\mathrm{Li}$ and Chen 2019). In this study, we have found that the fusaricidin fermentation broth of $P$. polymyxa WLY78 showed 64\% biocontrol efficacy against Fusarium head blight and improved wheat yields by $24 \%$. Our study suggests that fusaricidin has potential to be used in the control of Fusarium head blight.

In order to enhance fusaricidin production, we attempt to reveal the regulatory network of the fus gene transcription of
A

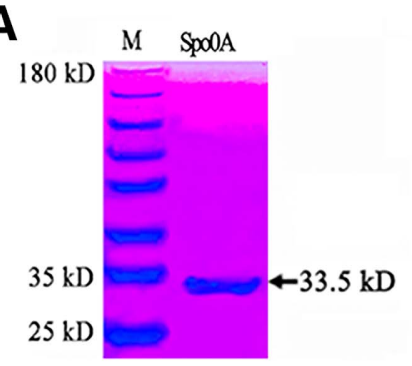

C $a b r B$ promoter sequence

GACATTCTTTGTCGTAATATGTCGAATTATAGTTTTTCGACATTTCCAAATCGACAAATT GAATAAATCATCAATAAGATAAGGCAATGCACATGAGTAAAGCTCTTTAATCTAAATACT TTTGCATAATCAGACTGTTACTACATTTTTTAGGTTGACTGTTTTGGGAATCGTTGGTAT AGTAAATATCAGAAAAGAGAAAATTTTGTCGAATCATGACACTTATATTATTTTAAACGG CGAGAGGAGCCTGATTTATT

D

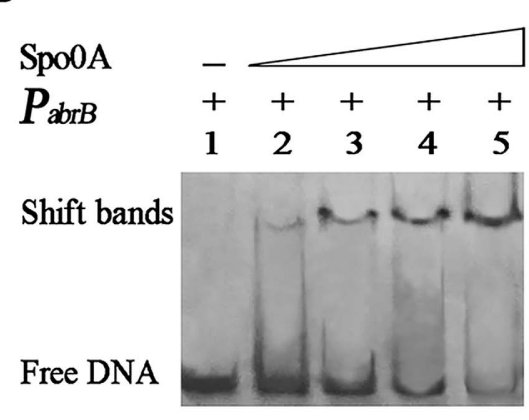

E

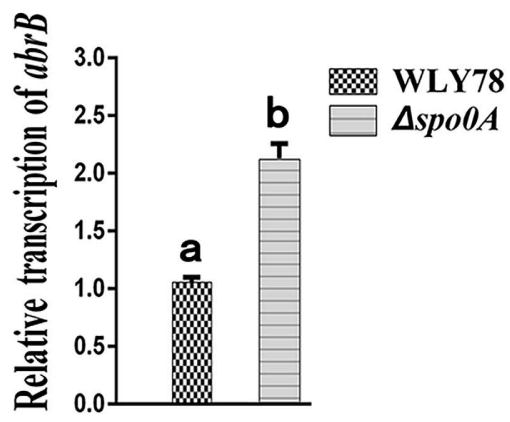

Fig. 5. Determination of Spo0A-binding site in the $a b r B$ promoter by electrophoretic mobility shift assay (EMSA) and effect of $s p o 0 A$ mutation on the abrB transcription. A, Sodium dodecyl sulfate-polyacrylamide gel electrophoresis analysis of the expressed and purified Paenibacillus polymyxa His ${ }^{-}$ Spo0A protein in Escherichia coli. B, EMSA analysis of the binding ability of Spo0A protein to the fus promoter region. Lanes 1 through 5 indicate increasing concentrations of Spo0A protein $(0,5,20,50,200 \mathrm{ng})$ and 0.1 pmol-labeled promoter DNA was used for each lane. C, Spo0A-binding sites in the promoter region of the $a b r B$ gene. D, Transcription levels of the $a b r B$ gene in $P$. polymyxa WLY78 and $\triangle$ spoOA mutant determined by quantitative reverse transcription-PCR. E. EMSA analysis of the binding ability of Spo0A protein to the $a b r B$ promoter region. Lanes 1 through 5 indicate increasing concentrations of SpoOA protein $(0,5,20,50,200 \mathrm{ng})$ and 0.1 pmol-labeled promoter DNA was used for each lane. Lower case letters indicate statistically significant difference $(P<0.05)$. 
$P$. polymyxa WLY78. Our studies have shown that AbrB as a transcription protein represses fusaricidin biosynthesis in $P$. polymyxa WLY78 by direct binding on the promoter region of the fus gene cluster, consistent with the results obtained in $P$. polymyxa SQR-21 (Li et al. 2013). We provided evidence suggesting that the regulator AbrB represses the transcription of the fus gene cluster by direct binding to the fus promoter, in which the sequences (5' -AATTTTAAAATAAATTTTGTGATTT-3') located from -136 to -112 bp relative to the transcription start site are required for this repression. Previous studies on the transcriptional properties of $P$. polymyxa SQR-21 inferred that the fus promoter region between -383 and -282 bp (relative to transcription start codon) is a binding region for some negative regulators ( $\mathrm{Li}$ et al. 2013). By comparing the AbrB-binding sequences in the fus promoter of $P$. polymyxa WLY78 with those in $B$. subtilis AbrB-dependent promoter region, A/T-rich sequences are evident. A relevant
TGGNA motif connected by A/T-rich sequences in AbrBbinding sites is identified in some genes of B. subtilis (Chumsakul et al. 2011; Xu and Strauch 1996). But neither the TGGNA motif nor a clear consensus DNA sequence is detected in the fus promoter region of $P$. polymyxa WLY78. Our results are consistent with the previous reports that $\mathrm{AbrB}$ does not recognize a clear consensus DNA sequence in B. subtilis (Olson et al. 2014). AbrB functions as a tetramer or dimer in DNA regulation scale, which means that multiple binding motifs for AbrB are possible (Sullivan et al. 2008).

Our mutation analyses have revealed that $P$. polymyxa Spo0A is required for efficient fusaricidin production of $P$. polymyxa WLY78. There is no 0A box (TGTCGAA) for binding of Spo0A in the fus promoter region, and EMSA also has shown that Spo0A does not bind to the fus promoter region, suggesting that the Spo0A regulates the fus transcription indirectly. However,

A

B
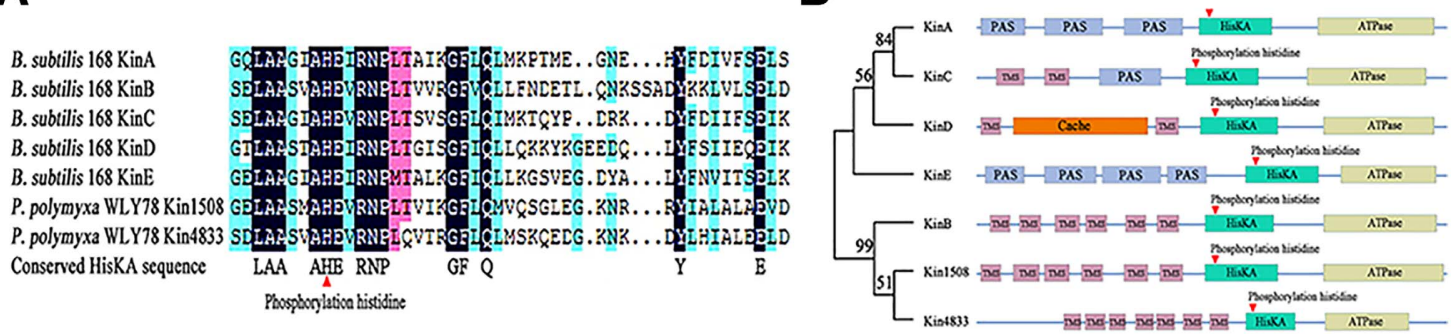

C

WLY78

$\Delta \operatorname{kin} 1508$

$\Delta$ kin 4833

$\Delta$ kin 1508

$\Delta$ kin4833

(kin1508)

(kin4833)

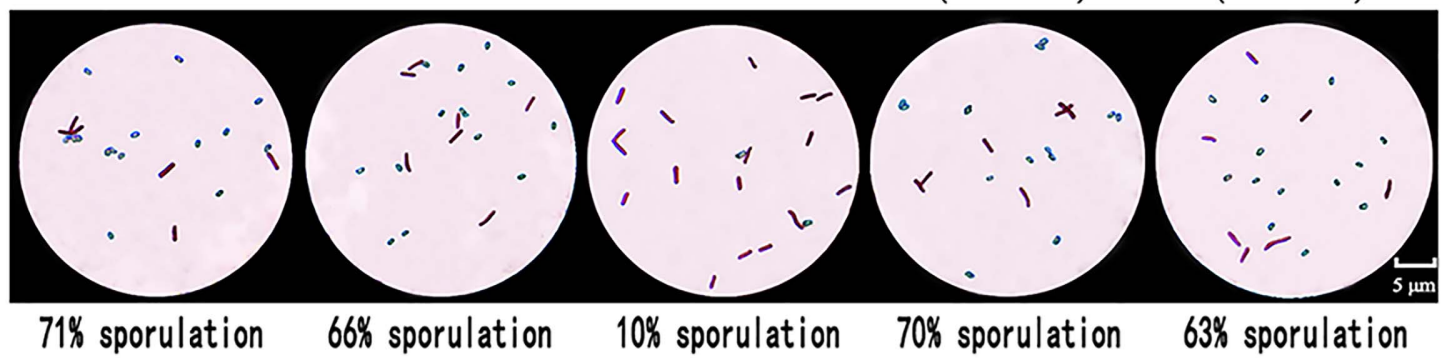

D

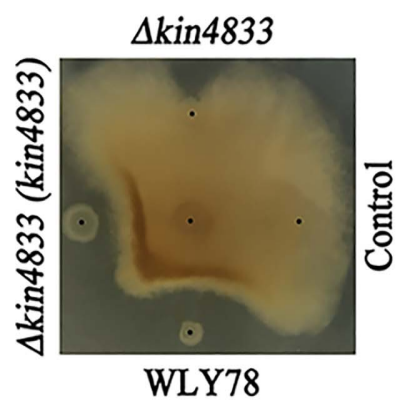

E

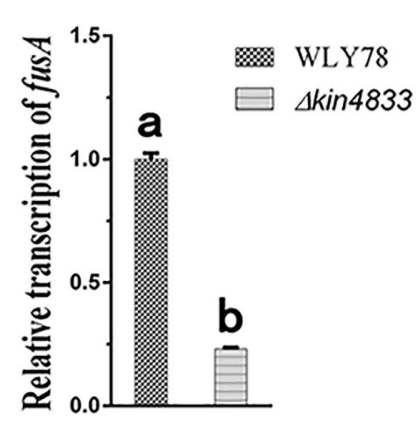

F

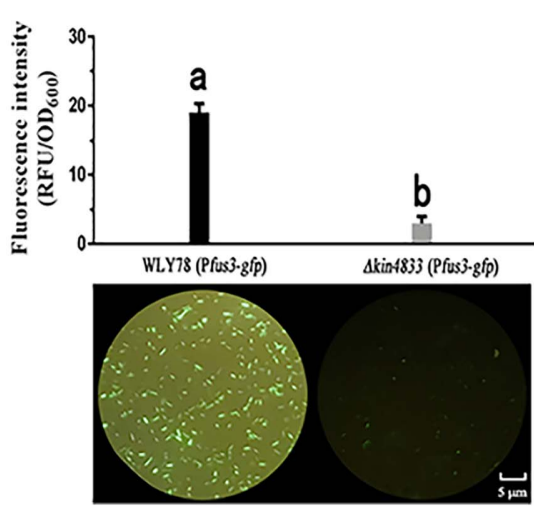

Fig. 6. Homology analysis of histidine kinases of Paenibacillus polymyxa WLY78 with those of Bacillus subtilis 168 and comparison of sporulation formation, antifungal activities, fusA transcription levels, fluorescence intensity of the Pfus3-gfp fusion among P. polymyxa WLY78, $\Delta$ kin1508, $\Delta k i n 4833$, and complementation strains. A, Alignment of histidine kinases (Kin1508 and Kin4833) of P. polymyxa WLY78 and histidine kinases (KinA, KinB, KinC, and KinD) of B. subtilis 168 by using the conserved sequence of HisKA domains. B, Phylogenetic tree and domain organization of $P$. polymyxa kinases (Kin1508 and Kin4833) and B. subtilis 168 kinases (KinA, KinB, KinC, KinD, and KinE). C, Sporulation frequencies of $P$. polymyxa WLY78, $\Delta$ kin1508, and $\Delta$ kin4833 and complementation strains $\Delta$ kin1508 (kin1508) and $\Delta$ kin4833 (kin4833). Rod cell in dark red is vegetative cell and spheroid cell in blue is spore. D, The inhibition activities against Fusarium asiaticum by $P$. polymyxa WLY78, $\Delta$ kin 4833 mutant, and complementation strain $\Delta$ kin4833 (kin4833). E, Transcription levels of the fusA gene in $P$. polymyxa WLY78 and $\Delta a b r B$ mutant determined by quantitative reverse transcription-PCR. F, Fluorescence microscope observation and fluorescence intensity of $P$. polymyxa WLY78 and $\Delta$ kin 4833 carrying the Pfus3-gfp fusion. Lower case letters indicate statistically significant difference $(P<0.05)$. 
there are two 0A boxes in the $a b r B$ promoter region of $P$. polymyxa WLY78, and EMSA has demonstrated that Spo0A directly binds to the $a b r B$ promoter region. qRT-PCR has shown that $a b r B$ expression is inhibited in the $\triangle$ spoOA mutant. The results suggest that $P$. polymyxa Spo0A indirectly activates fusaricidin production by directly repressing $a b r B$ expression, consistent with the findings that Spo0A negatively controlled $a b r B$ expression in B. subtilis 168 (Fujita et al. 2005; Strauch et al. 2007).

The activity of Spo0A is governed by a multicomponent phosphorylation in Bacillus spp. The phosphorylation of Spo0A $(\mathrm{SpoOA} \sim \mathrm{P})$ is controlled by five histidine kinases (KinA, KinB, $\mathrm{KinC}$, KinD, and KinE), each of which senses different signals of sporulation (Banse et al. 2011; Brunsing et al. 2005; Jiang et al. 2000; Zeriouh et al. 2014). Among these histidine kinases, KinA and KinB are thought to be the major kinases for initiating sporulation (Liu et al. 2018; Tojo et al. 2013). In this study, we screened the $P$. polymyxa WLY78 genome and found two putative histidine kinases, named Kin1508 and Kin4833. The P. polymyxa Kin1508 and Kin4833 exhibit 23 and $31 \%$ identity, respectively, with $\mathrm{KinB}$ of $B$. subtilis 168 at the amino-acid sequence level. Protein domain analysis has shown that each of the Kin1508 and Kin4833 proteins of P. polymyxa WLY78 has three domains, transmembrane, HisKA, and ATPase. A conserved histidine residue for auto-phosphorylation is found in the N-terminal domain of both Kin1508 and Kin4833 proteins. The structure characteristics of the Kin1508 and Kin4833 proteins suggest that they are the sensor kinases that carry out autophosphorylation and then transfer a phosphoryl group to a receptor. To investigate whether both $P$. polymyxa Kin 1508 and Kin4833 are involved in phosphorylation of Spo0A (Spo0A P), the sporulation frequencies are used as an indicator of the phosphorylation levels (Kiehler et al. 2017). The $\Delta$ kin4833 mutant showed significant reduction in the sporulation ability compared with $P$. polymyxa WLY78, and the complementation strain restored the sporulation defect, indicating that Kin 4833 may be involved in phosphorylation of SpoOA to initiate sporulation. Further, the antifungal ability and the transcription levels of fusA gene in the $\Delta$ kin 4833 mutant are much lower than those in wild type, suggesting that Kin4833 is positively correlated with fus transcription and is consistent with the results obtained in $\triangle$ spoOA mutant.

In B. subtilis, KinB or KinA, as the sensor kinase, carries out autophosphorylation and then transfers a phosphoryl group to SpoOF, the resulting SpoOF $\sim \mathrm{P}$, in turn, transfers the phosphoryl group to Spo0B. Finally, SpoOB $\sim \mathrm{P}$ transfers the phosphoryl group to Spo0A, and the resulting Spo0A P activates or represses gene expression (Tojo et al. 2013). However, there are no SpoOF and Spo0B and only the Spo0A protein is found in Clostridial genomes, suggesting that Clostridial species, the anaerobic relatives of Bacillus genus, do not use a multicomponent phosphorylation system but use a classical two-component module in which Spo0A is activated directly by a sensor kinase (Stephenson and Hoch 2002). We have found that, in addition to the $s p o 0 A$ gene, there are a $s p o O F$ and a $\triangle p o O B$ in the genome of $P$. polymyxa WLY78. Thus, we deduce that $P$. polymyxa Spo0A may be phosphorylated and activated by a multicomponent phosphorylation system composed of $\mathrm{Kin} 4833$ (KinB), Spo0B, and Spo0F, as $B$. subtilis did.

On the basis of our results and the previous findings obtained in B. subtilis, we propose a regulation mode of fusaricidin production of $P$. polymyxa WLY78. During nutrient limitation, Kin4833 (KinB) senses the specific signals and is auto-phosphorylated, yielding $\mathrm{KinB} \sim \mathrm{P}$. Then, $\mathrm{KinB} \sim \mathrm{P}$ transfers its phosphoryl group to $\mathrm{SpoOF}$, the resulting $\mathrm{SpoOF} \sim \mathrm{P}$, in turn, transfers the phosphoryl group to $\mathrm{SpoOB}$, and finally, $\mathrm{SpoOB} \sim \mathrm{P}$ transfers the phosphoryl group to Spo0A. The resulting SpoOA $\sim \mathrm{P}$ negatively regulates the expression of $\mathrm{AbrB}$ that represses the fus expression by binding to the fus promoter, in which the sequences $\left(5^{\prime}\right.$-AATTTTAAAA TAAATTTTGTGATTT- $3^{\prime}$ ) located from -136 to -112 bp relative to the transcription start site is required for this repression. Therefore, fusaricidin is produced with spore formation under nutrient limitation (Fig. 7). Under excess nutrient conditions, Kin4833 (KinB) is not auto-phosphorylated, and thus Spo0A is not phosphorylated to repress the $a b r B$ expression. Thus, AbrB binds to the fus promoter to inhibit fusaricidin biosynthesis. The signal transduction pathway by phosphorylation among $\mathrm{KinB}$, SpoOF, Spo0B, and Spo0A in fusaricidin synthesis of $P$. polymyxa WLY78 needs to be deeply studied by using the (r- $\left.{ }^{32} \mathrm{P}\right)$ ATP method described by Haydel et al. (1999) and Cui et al. (2011).

\section{MATERIALS AND METHODS}

Microorganisms, plasmids, and culture conditions.

The source of strains and plasmids are summarized in Supplementary Table S1. E. coli DH5 $\alpha$ was cultivated at $37^{\circ} \mathrm{C}$ in LuriaBertani (LB) broth for cloning of plasmids. E. coli BL21 (DE3) was cultivated at $30^{\circ} \mathrm{C}$ in LB broth for protein expression. P. polymyxa WLY78 was routinely cultivated at $30^{\circ} \mathrm{C}$ in $\mathrm{LB}$ medium. The synthetic medium, which was described by Katznelson and Lochhead (1944) and used for biosynthesis of polymyxin b by (Paulus and Gray 1964), was used for fusaricidin production. Pathogen $F$. asiaticum was cultivated at $28^{\circ} \mathrm{C}$ in potato dextrose agar (PDA) medium. Thermo-sensitive vector pRN5101 was used for gene deletion in $P$. polymyxa. Shuttle vector pHY300PLK was used for the complementation experiment and $g f p$ fusion. Vector pET-28b was used for expressing recombinant $\mathrm{His}_{6}$-tagged protein in E. coli BL21. When necessary, LB broth was solidified by adding $1.5 \%$ agar, and the antibiotics were added in the following concentrations: ampicillin, $100 \mu \mathrm{g} / \mathrm{ml}$; erythromycin, $5 \mu \mathrm{g} / \mathrm{ml}$; and tetracycline, $12.5 \mu \mathrm{g} / \mathrm{ml}$.

\section{Preparation of fusaricidin fermentation broth.}

$P$. polymyxa WLY78 was grown in LB liquid medium for overnight at $30^{\circ} \mathrm{C}$ with shaking at $200 \mathrm{rpm}$. Then, $500 \mathrm{ml}$ of liquid culture was transferred into a 7.5-liter fermenter filled with 5 liters of the synthetic medium (Paulus and Gray 1964).

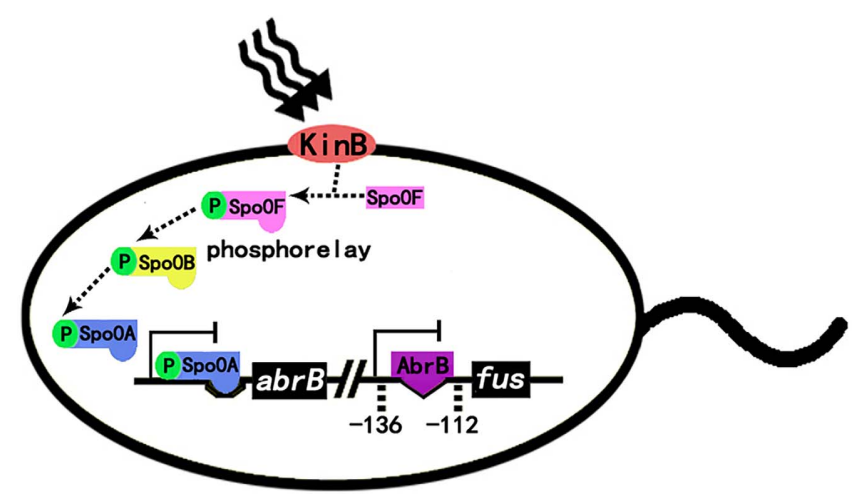

Fig. 7. The predicted regulatory network that controls fusaricidin production of Paenibacillus polymyxa WLY78. Kin4833 (KinB) senses the specific signals and is auto-phosphorylated, yielding $\mathrm{KinB} \sim \mathrm{P}$. Then, $\mathrm{KinB} \sim \mathrm{P}$ transfers its phosphoryl group to $\mathrm{Spo} 0 \mathrm{~F}$, the resulting $\mathrm{Spo} 0 \mathrm{~F} \sim \mathrm{P}$, in turn, transfers the phosphoryl group to Spo0B, and finally, Spo0B P transfers the phosphoryl group to Spo0A. The resulting Spo0A $\sim \mathrm{P}$ negatively regulates the expression of $\mathrm{AbrB}$ that represses the fus expression by binding to the fus promoter, in which the sequences ( $5^{\prime}$-AATTTTAAAATAAAT TTTGTGATTT $-3^{\prime}$ ) located from -136 to -112 bp relative to transcription start site is required for this repression. Dashed arrow indicates the signal transduction by phosphorylation that needs to be determined by experiments and the $\mathrm{T}$ bar indicates repressed gene expression that has been confirmed by our experiments. 
During the fermentation process, temperature was maintained at $30^{\circ} \mathrm{C}, \mathrm{pH}$ was controlled at 6.8 by the addition of $5 \mathrm{M}$ $\mathrm{KOH}$, and culture was continuously stirred at $200 \mathrm{rpm}$. The liquid culture was harvested after 3 days of fermentation and was then used for control of wheat Fusarium head blight. The bacterial cell density was about $1 \times 10^{9}$.

\section{Biological control of wheat Fusarium head blight in field trials.}

The field trials were conducted at the Wuqiao Experimental Station of China Agricultural University at Cangzhou, one of the most important wheat production areas in Hebei Province, China. This area had been cultivated with winter wheat-summer maize for many years. The areas were naturally infested by $F$. asiaticum. Winter wheat was sown in October and was harvested the following June. A randomized block design was adopted for experiments. Six plots, each of which was 40 square meters, were used for our experiment. Three plots were treated with fusaricidin fermentation broth, and another three plots were treated with tap water as control. In the early period of May, when wheat scab did not occur, $33 \mathrm{ml}$ of fusaricidin fermentation broth was diluted with tap water ( $\mathrm{vol} / \mathrm{vol}) 1: 10$ and was then applied to the aerial part of wheat seedlings via a sprayer in each plot. Tap water was used instead of fusaricidin fermentation broth in the control group. Crop management followed routine commercial practices. A total of 100 wheat seedlings from each plot were collected to investigate the disease severity at 28 days after treatment. The disease severity was scored using a grade value, denoting proportions of disease over the whole wheat ear area, where grade $0: 0 \%$, grade $1:<25 \%$, grade $2: 25$ to $50 \%$, grade 3: 50 to $75 \%$, grade 4: 75 to $100 \%$, grade 5:100\% (Landschoot et al. 2012). Then the disease index (DI) and control efficacy (CE) was computed, using the formula

$$
D I=\frac{\sum\left(g N_{g}\right)}{5 N_{t}},
$$

where $g$ is the grade value, $N_{g}$ is the number of the plants of the corresponding grade, and $N_{t}$ is the total number of plants in each group. Then, the CE was determined by the following formula.

$$
C E(\%)=\left[1-\frac{D I_{\text {treatment }}}{D I_{\text {control }}}\right] \times 100 \%
$$

To calculate the grain yield per hectare, the grains in three plots were harvest and weighed.

\section{Bioinformatics analysis of histidine kinases in $P$. polymyxa.}

A functional domain and homology search was performed, using the BLAST program. ClustalW was used for sequence alignment. The domain structures were predicted using HMMER.

\section{Construction of $\Delta k i n 1508, \Delta k i n 4833, \Delta s p o 0 A$, and}

\section{$\triangle a b r B$ mutants and their complementation strains.}

Four in-frame deletion mutants, $\Delta$ kin $1508, \Delta$ kin $4833, \Delta s p o 0 A$, and $\triangle a b r B$, were constructed by a homologous recombination method. Briefly, the upstream $(1 \mathrm{~kb})$ and downstream fragments (1 kb) flanking the coding region of kin1508, kin4833, spo0A, and $a b r B$ were PCR-amplified from the genomic DNA of $P$. polymyxa WLY78. Briefly, the two fragments flanking each coding region of kin1508, kin4833, spo0A, and $a b r B$ were assembled into the BamHI-digested pRN5101 vector, yielding the four recombinant plasmids pRN-kin1508, pRN-kin4833, pRN-spo0A, and $\mathrm{pRN}-a b r B$, respectively. Subsequently, each recombinant plasmid was transformed into $P$. polymyxa WLY78. For selecting the $\Delta k \operatorname{kin} 1508, \Delta k i n 4833, \Delta s p o 0 A$, and $\Delta a b r B$ mutants, the transformants were cultivated at $39^{\circ} \mathrm{C}$ and were identified by PCR according to the methods described by Li and Chen (2019). The primers for PCR are listed in Supplementary Table S2.

For complementation of $\Delta$ kin1508, $\Delta$ kin4833, $\Delta$ spo0A, and $\triangle a b r B$ mutants, four DNA fragments carrying the gene open reading frames and their own promoters, Pkin1508 (1,478 bp), Pkin4833 (2,022 bp), Pspo0A (1,020 bp), and PabrB (515 bp) were PCR-amplified, respectively. Then, each fragment was assembled into the EcoRI-digested pHY300PLK vector and then transformed into $P$. polymyxa WLY78, respectively. The PCR primers are listed in Supplementary Table S2. The nucleotide sequence of kin1508, kin4833, spo0A, and $a b r B$ in $P$. polymyxa WLY78 were deposited in GenBank under accession numbers MT096381, MT096382, MT096380, and MT096379, respectively.

\section{Antifungal activity assays.}

To test the antifungal activity of $P$. polymyxa WLY78 and its mutant strains, the inhibition zone of the strain against the fungi $F$. asiaticum was recorded. Briefly, the $F$. asiaticum was inoculated onto the center of PDA medium. Then, $1 \mu \mathrm{l}$ of $P$. polymyxa cell suspensions $\left(10^{7} \mathrm{CFU} / \mathrm{ml}\right)$ and mutant cell suspensions $\left(10^{7} \mathrm{CFU} / \mathrm{ml}\right)$ were respectively inoculated around the fungi at a distance of $2 \mathrm{~cm}$, with $1 \mu \mathrm{l}$ of sterilized water as a negative control (Li and Chen 2019). All the plates were cultured at $28^{\circ} \mathrm{C}$ for 4 days. Then the inhibition effect on fungi hyphae growth was recorded.

\section{Spore assays.}

To evaluate the sporulation efficiency of $P$. polymyxa WLY78 and kinase mutant, the ratio of spores was calculated as follows. One milliliter of overnight bacterial culture grown in LB broth was inoculated into $20 \mathrm{ml}$ of the synthetic medium (Paulus and Gray,1964) and was grown at $37^{\circ} \mathrm{C}$ with shaking at $220 \mathrm{rpm}$ for 3 days. Then the cells were collected and were stained using Schaeffer-Fulton's method, after which they were observed under an optical microscope (Mormak and Casida 1985).

\section{RNA preparation and qRT-PCR assay.}

To compare the relative expression level of fusA between $P$. polymyxa WLY78 and each mutant, qRT-PCR analysis was performed as follows. After cultivation for 3 days, $50 \mathrm{ml}$ of culture were harvested and rapidly frozen in liquid nitrogen. Total RNA was extracted by RNAiso Plus (TaKaRa) and $1 \mu \mathrm{g}$ of total RNA was converted into cDNA with a reverse transcription reagent kit (TaKaRa). 16S RNA was used as an internal reference. The qRT-PCR procedure was conducted as described previously ( $\mathrm{Li}$ et al. 2015). The relative transcription level was calculated using the $\Delta^{\Delta \mathrm{CT}}$ method (Livak and Schmittgen 2001). The primers are listed in Supplementary Table S3.

\section{Expression and purification of Spo0A and AbrB proteins in $E$. coli.}

The $s p o 0 A$ and $a b r B$ genes were PCR-amplified from the genomic DNA of $P$. polymyxa WLY78. These PCR products were cloned into $\mathrm{pET}-28 \mathrm{~b}$ to construct tagged proteins with $\mathrm{His}_{6}$-tag at the $\mathrm{N}$ terminal and were then transformed into $E$. coli BL21 (DE3). The recombinant E. coli strains were cultivated in LB broth with $50 \mu \mathrm{g}$ of kanamycin per milliliter until the optical density at $600 \mathrm{~nm}\left(\mathrm{OD}_{600}\right)=0.5$, when $0.2 \mathrm{mM}$ IPTG was added and the cells were incubated at $30^{\circ} \mathrm{C}$ for $4 \mathrm{~h}$. Then the cells were collected in a lysis buffer $\left(50 \mathrm{mM} \mathrm{NaH}_{2} \mathrm{PO}_{4}, 300 \mathrm{mM} \mathrm{NaCl}, 10 \mathrm{mM}\right.$ imidazole) and sonication on ice. Recombinant $\mathrm{His}_{6}$-tagged proteins in the supernatant were purified on $\mathrm{Ni}_{2}-\mathrm{NTA}$ resin (Qiagen), according to the manufacturer instructions. Proteins eluted with $250 \mathrm{mM}$ imidazole were dialyzed into binding buffer 
(20 mM HEPES, pH 7.6, $1 \mathrm{mM}$ EDTA, $10 \mathrm{mM}\left(\mathrm{NH}_{4}\right)_{2} \mathrm{SO}_{4}, 1$ $\mathrm{mM}$ dithiothreitol, $0.2 \%$ Tween 20 , and $30 \mathrm{mM} \mathrm{KCl}$ ) for EMSAs. The primers are listed in Supplementary Table S4.

\section{EMSA.}

EMSAs were performed using the DIG gel shift kit (Roche), according to the manufacture instructions. The promoter fragments of the fus operon were synthesized by Sangon Biotech Co., Ltd. Two DNA fragments corresponding to the sequences of the first strand and the complementary DNA strand were synthesized. The two strands were annealed and then labeled at the $3^{\prime}$ end with digoxigenin using terminal transferase and were used as probes. Each binding reaction $(20 \mu \mathrm{l})$ consisted of $1 \mu \mathrm{g}$ poly [d(A-T)], $0.3 \mathrm{nM}$ labeled probe, and various concentration of purified His $_{6}$-protein in the binding buffer. Binding reaction was performed at $25^{\circ} \mathrm{C}$ for $30 \mathrm{~min}$ and was then analyzed by electrophoresis, using native $5 \%$ polyacrylamide gel run with $0.5 \times$ Tris-borateEDTA as running buffer and electrophoretically transferred to a positively charged nylon membrane at $4^{\circ} \mathrm{C}$ (GE Healthcare). Labeled DNAs were detected by chemiluminescence, according to manufacturer protocol, and recorded on X-ray film. The primers are listed in Supplementary Table S5.

\section{Transcription start site identification.}

The 5' RACE assay was performed, using the SMARTer RACE cDNA amplification kit (Clontech). Gene-specific primers are listed in Supplementary Table S5. The PCR fragment obtained from 5' RACE was inserted into the pMD18-T vector and individual clones were selected for DNA sequencing.

\section{Pfus-gfp fusion plasmid construction and GFP activity assay.}

To evaluate the fus expression level, various different lengths of the fus promoter region were fused to the $g f p$ coding region (Pfus-gfp fusions) (Fig. 3A). Briefly, eight fus promoter DNA fragments (from $-336,-236,-136,-111,-86,-61,-36$, and -1 to +26 relative to the transcription start site) were respectively amplified from the genomic DNA of $P$. polymyxa WLY78, using the primers listed in Supplementary Table S6. The coding region of $g f p$ was PCR-amplified with primers from the plasmid pGFP4412. Each fus promoter fragment and $g f p$ fragment were assembled into the vector pHY300PLK digested by BamHI, yielding eight Pfus-gfp fusion plasmids Then, each of Pfus-gfp fusion plasmid was transformed into $P$. polymyxa WLY78, $\Delta$ kin4833, $\Delta s p o 0 A, \Delta a b r B$, and the recombinant E. coli BL21 carrying the $a b r B$ gene in pET-28b vector that could overexpress AbrB when induced by $0.2 \mathrm{mM}$ IPTG. The transformants were cultured overnight in LB broth with $12.5 \mu \mathrm{g}$ of tetracycline per milliliter at $37^{\circ} \mathrm{C}$ with shaking at $200 \mathrm{rpm}$. The $\mathrm{OD}_{600}=1$ of the bacterial suspension was adjusted. A drop of the bacterial suspension was taken to observe the green fluorescence of these transformants carrying the Pfus-gfp fusion plasmids by fluorescence microscope (Olympus BX60). Meanwhile, $200 \mu \mathrm{l}$ of suspension was filled into the 96-well cell plate for relative fluorescence unit assay by the multidetection microplate fluorescence reader (BioTek FLX800). The fluorescence intensity $(\mathrm{FI})$ was calculated by the following formula:

$$
\mathrm{FI}=\frac{\text { Relative fluorescence unit }(\mathrm{RFU})}{\mathrm{OD}_{600}}
$$

\section{Statistical analysis.}

All the experiments were repeated three times with a similar result. The significant difference $(P<0.01)$ of data were analyzed by one-way analysis of variance with Duncan's multiplerange test, using SPSS version 22 statistical software.

\section{ACKNOWLEDGMENTS}

Thanks to the Wuqiao Experimental Station of China Agricultural University at Cangzhou in Hebei Province of China for providing experimental support.

\section{AUTHOR-RECOMMENDED INTERNET RESOURCES}

Basic Local Alignment Search Tool (BLAST):

https://blast.ncbi.nlm.nih.gov/Blast.cgi

HMMERweb server: https://www.ebi.ac.uk/Tools/hmmer

\section{LITERATURE CITED}

Bailey, T. L., Boden, M., Buske, F. A., Frith, M., Grant, C. E., Clementi, L., Ren, J., Li, W. W., and Noble, W. S. 2009. MEME SUITE: Tools for motif discovery and searching. Nucleic Acids Res. 37:W202-208.

Banse, A. V., Hobbs, E. C., and Losick, R. 2011. Phosphorylation of Spo0A by the histidine kinase KinD requires the lipoprotein med in Bacillus subtilis. J. Bacteriol. 193:3949-3955.

Beatty, P. H., and Jensen, S. E. 2002. Paenibacillus polymyxa produces fusaricidin-type antifungal antibiotics active against Leptosphaeria maculans, the causative agent of blackleg disease of canola. Can. J. Microbiol. 48:159-169.

Bobay, B. G., Benson, L., Naylor, S., Feeney, B., Clark, A. C., Goshe M. B., Strauch, M. A., Thompson, R., and Cavanagh, J. 2004. Evaluation of the DNA binding tendencies of the transition state regulator AbrB. Biochemistry 43:16106-16118.

Bochmann, S. M., Spieß, T., Kötter, P., and Entian, K. D. 2015. Synthesis and succinylation of subtilin-like lantibiotics are strongly influenced by glucose and transition state regulator AbrB. Appl. Environ. Microbiol. 81:614-622.

Brunsing, R. L., La Clair, C., Tang, S., Chiang, C., Hancock, L. E., Perego, M., and Hoch, J. A. 2005. Characterization of sporulation histidine kinases of Bacillus anthracis. J. Bacteriol. 187:6972-6981.

Chumsakul, O., Takahashi, H., Oshima, T., Hishimoto, T., Kanaya, S., Ogasawara, N., and Ishikawa, S. 2011. Genome-wide binding profiles of the Bacillus subtilis transition state regulator AbrB and its homolog Abh reveals their interactive role in transcriptional regulation. Nucleic Acids Res. 39:414-428.

Cui, Y. H., Tu, R., Wu, L., Hong, Y. Y., and Chen, S. 2011. A hybrid two-component system protein from Azospirillum brasilense Sp7 was involved in chemotaxis. Microbiol. Res. 166:458-467.

Deng, Y., Lu, Z., Lu, F., Zhang, C., Wang, Y., Zhao, H., and Bie, X. 2011. Identification of LI-F type antibiotics and di-n-butyl phthalate produced by Paenibacillus polymyxa. J. Microbiol. Methods 85:175-182.

Fisher, S. H., Strauch, M. A., Atkinson, M. R., and Wray, L. V., Jr. 1994. Modulation of Bacillus subtilis catabolite repression by transition state regulatory protein AbrB. J. Bacteriol. 176:1903-1912.

Frisby, D., and Zuber, P. 1991. Analysis of the upstream activating sequence and site of carbon and nitrogen source repression in the promoter of an early-induced sporulation gene of Bacillus subtilis. J. Bacteriol. 173:7557-7564.

Fujita, M., González-Pastor, J. E., and Losick, R. 2005. High- and lowthreshold genes in the Spo0A regulon of Bacillus subtilis. J. Bacteriol. 187:1357-1368

Haron, M. H., Avula, B., Shi, Q., Li, X. C., Ashfaq, M. K., Bae, J. Y., Guan, S., Hinchee, M., Khan, I. A., and Khan, S. I. 2019. Quantitative determination and pharmacokinetic study of fusaricidin $\mathrm{A}$ in mice plasma and tissues using ultra-high performance liquid chromatography-tandem mass spectrometry. J. Pharm. Biomed. Anal. 170:187-192.

Haydel, S. E, Dunlap, N. E, and Benjamin, W. H. 1999. In vitro evidence of two-component system phosphorylation between the Mycobacterium tuberculosis TrcR/TrcS proteins. Microb. Pathogenesis 26:195-206.

Jeong, H., Choi, S. K., Ryu, C. M., and Park, S. H. 2019. Chronicle of a soil bacterium: Paenibacillus polymyxa E681 as a tiny guardian of plant and human health. Front. Microbiol. 10:467.

Jiang, M., Shao, W., Perego, M., and Hoch, J. A. 2000. Multiple histidine kinases regulate entry into stationary phase and sporulation in Bacillus subtilis. Mol. Microbiol. 38:535-542.

Kajimura, Y., and Kaneda, M. 1996. Fusaricidin A, a new depsipeptide antibiotic produced by Bacillus polymyxa KT-8. Taxonomy, fermentation, isolation, structure elucidation and biological activity. J. Antibiot. (Tokyo) 49:129-135.

Katznelson, H., and Lochhead, A. G. 1944. Studies with Bacillus polymyxa: III. Nutritional requirements. Can. J. Res. 22C:273-279. 
Kaur, S., Balakrishnan, R., and Jayaraman, K. 1978. The correlation between antibiotic synthesis, transcription and sporulation in Bacillus polymyxa. Biochem. Biophys. Res. Commun. 81:50-57.

Kiehler, B., Haggett, L., and Fujita, M. 2017. The PAS domains of the major sporulation kinase in Bacillus subtilis play a role in tetramer formation that is essential for the autokinase activity. MicrobiologyOpen 6:e0481.

Kim, H. R., Park, S. Y., Kim, S. B., Jeong, H., Choi, S. K., and Park, S. H. 2014. Inactivation of the phosphoglucomutase gene pgm in Paenibacillus polymyxa leads to overproduction of fusaricidin. J. Ind. Microbiol. Biotechnol. 41:1405-1414.

Landschoot, S., Waegeman, W., Audenaert, K., Vandepitte, J., Haesaert, G., and De Baets, B. 2012. Toward a reliable evaluation of forecasting systems for plant diseases: A case study using Fusarium head blight of wheat. Plant Dis. 96:889-896.

Lee, S. H., Cho, Y. E., Park, S.-H., Balaraju, K., Park, J. W., Lee, S. W., and Park, K. 2013. An antibiotic fusaricidin: A cyclic depsipeptide from Paenibacillus polymyxa E681 induces systemic resistance against Phytophthora blight of red-pepper. Phytoparasitica 41:49-58.

Li, S., Zhang, R., Wang, Y., Zhang, N., Shao, J., Qiu, M., Shen, B., Yin, X., and Shen, Q. 2013. Promoter analysis and transcription regulation of fus gene cluster responsible for fusaricidin synthesis of Paenibacillus polymyxa SQR-21. Appl. Microbiol. Biotechnol. 97:9479-9489.

Li, Y., and Chen, S. 2019. Fusaricidin produced by Paenibacillus polymyxa WLY78 induces systemic resistance against Fusarium wilt of cucumber. Int. J. Mol. Sci. 20:5240.

Li, Y., Gu, Y., Li, J., Xu, M., Wei, Q., and Wang, Y. 2015. Biocontrol agent Bacillus amyloliquefaciens LJ02 induces systemic resistance against cucurbits powdery mildew. Front. Microbiol. 6:883.

Liu, J., Tan, K., and Stormo, G. D. 2003. Computational identification of the Spo0A-phosphate regulon that is essential for the cellular differentiation and development in gram-positive spore-forming bacteria. Nucleic Acids Res. 31:6891-6903.

Liu, W., He, Z., Gao, F., Yan, J., and Huang, X. 2018. Sensor kinase KinB and its pathway-associated key factors sense the signal of nutrition starvation in sporulation of Bacillus subtilis. MicrobiologyOpen 7:e00566.

Livak, K. J., and Schmittgen, T. D. 2001. Analysis of relative gene expression data using real-time quantitative PCR and the $2^{(-\Delta \Delta} \mathrm{C}(\mathrm{T})$ method. Methods 25:402-408.

Ma, Z., Xie, Q., Li, G., Jia, H., Zhou, J., Kong, Z., Li, N. and Yuan, Y. 2020. Germplasms, genetics and genomics for better control of disastrous wheat Fusarium head blight. Theor. Appl. Genet. 133:15411568.

Marahier, M. A., Nakano, M. M., and Zuber, P. 1993. Regulation of peptide antibiotic production in Bacillus. Mol. Microbiol. 7:631-636.

Mormak, D. A., and Casida, L. E. 1985. Study of Bacillus subtilis endospores in soil by use of a modified endospore stain. Appl. Environ. Microbiol. 49:1356-1360.

Nandy, S. K., and Venkatesh, K. V. 2008. Effect of carbon and nitrogen on the cannibalistic behavior of Bacillus subtilis. Appl. Biochem. Biotechnol. 151:424-432.

Nefelova, N. V., Filippova, M. S., and Egorov, N. S. 1980. [Effect of polymyxins on Bacillus polymyxa sporogenesis]. Mikrobiologiia 49:294-297.
Olson, A. L., Tucker, A. T., Bobay, B. G., Soderblom, E. J., Moseley, M. A., Thompson, R. J., and Cavanagh, J. 2014. Structure and DNA-binding traits of the transition state regulator AbrB. Structure 22:1650-1656.

Park, S. Y., Choi, S. K., Kim, J., Oh, T. K., and Park, S. H. 2012. Efficient production of polymyxin in the surrogate host Bacillus subtilis by introducing a foreign $e c t B$ gene and disrupting the $a b r B$ gene. Appl. Environ. Microbiol. 78:4194-4199.

Paulus, H., and Gray, E. 1964. The biosynthesis of polymyxin B by growing cultures of Bacillus polymyxa. J. Biol. Chem. 239:865-871.

Perego, M., Cole, S. P., Burbulys, D., Trach, K., and Hoch, J. A. 1989. Characterization of the gene for a protein kinase which phosphorylates the sporulation-regulatory proteins Spo0A and SpoOF of Bacillus subtilis. J. Bacteriol. 171:6187-6196.

Reimann, M., Sandjo, L. P., Antelo, L., Thines, E., Siepe, I., and Opatz, T. 2017. A new member of the fusaricidin family-structure elucidation and synthesis of fusaricidin E. Beilstein J. Org. Chem. 13:1430-1438.

Stephenson, K., and Hoch, J. A. 2002. Evolution of signalling in the sporulation phosphorelay. Mol. Microbiol. 46:297-304.

Strauch, M., Webb, V., Spiegelman, G., and Hoch, J. A. 1990. The SpoOA protein of Bacillus subtilis is a repressor of the $a b r B$ gene. Proc. Natl. Acad. Sci. U.S.A. 87:1801-1805.

Strauch, M. A. 1995a. Delineation of AbrB-binding sites on the Bacillus subtilis spoOH, $k i n B, f t s A Z$, and $p b p E$ promoters and use of a derived homology to identify a previously unsuspected binding site in the bsuB1 methylase promote. J. Bacteriol. 177:6999-7002.

Strauch, M. A. 1995b. In vitro binding affinity of the Bacillus subtilis AbrB protein to six different DNA target regions. J. Bacteriol. 177:4532-4536.

Strauch, M. A., Bobay, B. G., Cavanagh, J., Yao, F., Wilson, A., and Le Breton, Y. 2007. Abh and AbrB control of Bacillus subtilis antimicrobial gene expression. J. Bacteriol. 189:7720-7732.

Strauch, M. A., Spiegelman, G. B., Perego, M., Johnson, W. C., Burbulys, D., and Hoch, J. A. 1989. The transition state transcription regulator $a b r B$ of Bacillus subtilis is a DNA binding protein. EMBO J. 8:1615-1621.

Sullivan, D. M., Bobay, B. G., Kojetin. D. J., Thompson, R. J., Rance, M. Strauch, M. A., and Cavanagh, J. 2008. Insights into the nature of DNA binding of AbrB-like transcription factors. Structure 16:1702-1713.

Tojo, S., Hirooka, K., and Fujita, Y. 2013. Expression of kinA and kinB of Bacillus subtilis, necessary for sporulation initiation, is under positive stringent transcription control. J. Bacteriol. 195:1656-1665.

Vater, J., Niu, B., Dietel, K., and Borriss, R. 2015. Characterization of novel fusaricidins produced by Paenibacillus polymyxa-M1 using MALDITOF mass spectrometry. J. Am. Soc. Mass Spectrom. 26:1548-1558.

$\mathrm{Xu}, \mathrm{K}$, and Strauch, M. A. 1996. In vitro selection of optimal AbrBbinding sites: comparison to known in vivo sites indicates flexibility in AbrB binding and recognition of three-dimensional DNA structures. Mol. Microbiol. 19:145-158.

Zeriouh, H., de Vicente, A., Pérez-García, A., and Romero, D. 2014. Surfactin triggers biofilm formation of Bacillus subtilis in melon phylloplane and contributes to the biocontrol activity. Environ. Microbiol. 16:2196-2211.

Zhao, H., Msadek, T., Zapf, J., Madhusudan, Hoch, J. A., and Varughese, K. I. 2002. DNA complexed structure of the key transcription factor initiating development in sporulating bacteria. Structure 10:1041-1050. 